\title{
Empiricism and Intelligent Design I: Three Empiricist Challenges
}

\author{
Sebastian Lutz*
}

Draft: $2011-05-12$

\begin{abstract}
Due to the logical relations between theism and intelligent design (ID), there are two challenges to theism that also apply to ID. In the falsifiability challenge, it is charged that theism is compatible with every observation statement and thus asserts nothing. I argue that the contentious assumptions of this challenge can be avoided without loss of precision by charging theism (and thus ID) directly with the lack of observational assertions. In the translatability challenge, it is charged that theism can be translated into a (non-theistic) set of observation statements without loss of cognitive content. I argue that the contentious assumptions of this challenge are avoided by the related charge that the (non-theistic) evolutionary theory makes all the observational assertions of ID, while the converse does not hold. Elliott Sober has argued that ID meets the falsifiability challenge, but, since it makes almost no observational assertions, is not testable. I point out two problems with Sober's argument and show that ID is both deductively and probabilistically testable. Sober's argument, I suggest, inconsistently combines the modified falsifiability challenge with the modified translatability challenge. If his claims about ID's observational assertions are true, however, ID succumbs to the modified translatability challenge.
\end{abstract}

Keywords: empiricism; empirical significance; falsifiability; empirical content; testability; intelligent design; theism

\section{Contents}

1 Introduction 2

2 The falsifiability challenge 3

3 The translatability challenge $\quad 6$

4 The testability challenge $\quad 8$

5 The testability of ID 9

5.1 The deductive testability of ID $\ldots \ldots \ldots \ldots \ldots \ldots \ldots \ldots \ldots$

5.2 The contrastive testability of ID $\ldots \ldots \ldots \ldots \ldots \ldots \ldots \ldots \ldots \ldots \ldots \ldots$

6 The three challenges to intelligent design 11

*Theoretical Philosophy Unit, Utrecht University. sebastian.lutz@gmx.net. Many thanks to Thomas Müller and Elliott Sober for helpful comments and discussions. 


\section{Introduction}

The current discussion about the theory of intelligent design (ID) as a competitor of evolutionary theory (ET) is logically related to the discussion about theism and its empirical significance. This is because the statement 'God exists' may be translated as '[T] here exists necessarily a person without a body (i. e., a spirit) who necessarily is eternal, perfectly free, omnipotent, omniscient, perfectly good, and the creator of all things' (Swinburne 2004, 7), ${ }^{1}$ which entails but is not entailed by 'An intelligent designer exists'. Analogously, 'God created the biological organisms' entails but is not entailed by 'An intelligent designer created the biological organisms'.

That God is a specific designer is fairly uncontroversial. Mackie $(1982,1)$, for example, uses Swinburne's definition verbatim, and Sobel $(2004$, \$6) suggests that the central properties of God according to the "common conception of traditional theology" are "omnipotence, omniscience, perfect goodness, and being the Creator and Sustainer of the universe". ${ }^{2}$ An exception is Tooley $(1975,483)$, who defines God as "the one person who, though he can act within the world and can communicate with man, is neither dependent upon the world nor simply a part of it, but rather transcends the realm of human existence, and who, in addition, is morally perfect, omnipotent, omniscient, eternal, and incorporeal". Since Tooley does not define God to actually act or have acted in this world, according to his definition the existence of God arguably does not entail the existence of a designer. Of course, the statement that God created the biological organisms still entails that an intelligent designer created the biological organisms. In the following, the God of theism is always assumed to be a designer.

Many proponents of ID claim that the adaptations of biological organisms are evidence for the existence of an intelligent designer. This inference is weaker than the classic version of the teleological argument for the existence of God, also called the argument for (or to) design or, somewhat misleadingly, the argument from design (Mackie 1982, 133). This version, by Paley (1802), relies mostly on the adaptations of biological organisms as evidence, but concludes both that an intelligent designer exists (Paley 1802, \$XXIII) and that the designer has the properties of God (Paley

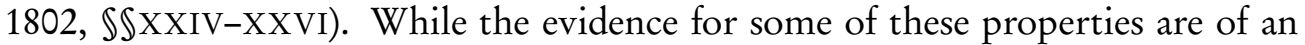
astronomical or chemical nature (Paley 1802, $\$ \$$ XXIf), the conclusions that Paley draws from the biological evidence alone go far beyond the existence of some designer.

Since Paley's conclusion is logically stronger than the conclusion of the ID argument, it is in greater danger of disconfirmation. The omnipotence and benevolence of God, for example, are in tension with the existence of evil (Mackie 1982, \$9; Sobel 2004, \$XII) and the maladaptations found in biological organisms (Mackie 1982, 138; Sobel 2004, \$7.1). The existence of an intelligent designer is not disconfirmed by such evidence, since a not further specified intelligent designer may fall short in power or benevolence.

On the other hand, there are two challenges to theism that are at least as and generally more urgent for logically weaker theories, and thus for ID. According to the falsifiability challenge, theism makes no assertions whatsoever because it is compatible

\footnotetext{
${ }^{1}$ With a reference to his discussion of the trinity, Swinburne adds in a footnote: "In understanding God as a person, while being fair to the Judaic and Islamic view of God, I am oversimplifying the Christian view."

${ }^{2}$ Nielsen $(1966,13)$ lists further definitions according to which God is a designer.
} 
with any consistent observation statement. According to the translatability challenge, there is a set of observation statements with the same cognitive content as theism, and since observation statements cannot contain references to God, any such reference is devoid of cognitive content.

Even assuming that theism is intended to be an empirical theory, it does not have to meet the challenges in their current form, for both rely on assumptions that are controversial, to put it mildly. In $\$ 2$, I will therefore review the falsifiability challenge and argue that its controversial assumptions can be avoided if theistic utterances are directly charged with lack of observational assertions rather than relying for the charge on their lack of falsifiability. I will review the translatability challenge in $\$ 3$ and argue that its problematic assumptions are avoided by a related challenge, according to which theism cannot replace a competing non-theistic theory if the competing theory has not been disconfirmed, and makes all the observational assertions of theism, but not vice versa. ID then faces both of these challenges as well.

In a different approach to the falsifiability challenge, Elliot Sober (1999, 2007, 2008) argues that for probabilistic theories, the criterion of falsifiability should be replaced by a new criterion of what I will call 'contrastive testability' $(\mathbb{S} 4)$. He then charges ID with a lack of testability. In $\$ 5.1$, I show that according to Sober's own claims, ID is testable because it is falsifiable. I will then argue that Sober's argument for ID's lack of contrastive testability is invalid, and that ID is in fact contrastively testable (\$5.2). Sober's challenge to ID, I suggest, inconsistently combines the modified falsifiability challenge with the modified translatability challenge. If his claims about ID's observational assertions are true, however, ID succumbs to the modified translatability challenge $(\$ 6)$.

\section{The falsifiability challenge}

The falsifiability challenge (the name is due to Tooley 1975,485 ) is motivated by the impression that theism has suffered the "death by a thousand qualifications", where a "fine brash hypothesis" is weakened until it fails to assert anything (Flew 1950, 258). Statements like 'God created the world' and 'God loves us as a father loves his children' (Flew 1950, 258), for example, seem to assert, among other things, that there are no maladaptations in organisms and nothing bad will ever happen to us (unless, maybe, we deserve it). But even though these assertions are false, many theists do not give up the original statements, and rather claim that God's creation and love differ from human creations and love. These modifications qualify the original statements to an extent that it becomes unclear whether the statements assert anything at all.

For Flew $(1950,259)$, this suggests a challenge to his fellow disputants:

I therefore put to the succeeding symposiasts the simple central question, 'What would have to occur or to have occurred to constitute for you a disproof of the love of, or of the existence of, God?'

Flew $(1950,258 \mathrm{f})$ justifies the challenge by arguing that to assert a statement is to deny its negation, so that someone who does not deny any statements also does not assert any.

Nielsen $(1966,15)$ points out that every statement meets the falsifiability challenge if there is no restriction on which statements one may deny, and suggests that Flew 
has to assume a restriction to "non-religious, straightforwardly empirical, factual statements". Flew $(1975,274)$ claims that Nielsen's restriction is too exclusive, and suggests a restriction to "anything which happens or which conceivably might happen in the ordinary world". This restriction is arguably too vague, since one may think, pace Tooley $(1975,483)$, that God exists in the ordinary world. I will just call the statements that the restriction allows 'observation statements', which is traditional. Their exact nature will not be important in the following, although Nielsen's remarks may serve as a guide.

Thus the falsifiability challenge rests on the assumption that a theory makes an assertion only if it is incompatible with an observation statement. But this is debatable because some assertions may just not be observational, and it is manifestly false for probabilistic theories. Sober $(2008,130$, cf. 151) gives the example of the statement that "a coin has a probability of .5 of landing heads each time it is tossed". This is an observational assertion, albeit a probabilistic one, and is compatible with any finite series of observations of coin tosses. Thus there is no reason to assume that only falsifiable theories make observational assertions, and no reason why a theory would have to meet the falsifiability challenge.

Tooley $(1975$, III) suggests to modify the falsifiability challenge so that proponents of a theory are not challenged to show its falsifiability, but rather its testability, that is, its confirmability or disconfirmability, where confirmation and disconfirmation rely on some concept of induction to be specified. But this strategy confuses an indicator (falsifiability) with the property it indicates (making assertions). Generalizing falsifiability to testability makes sense only if this at the same time generalizes the criterion from an indicator of theories that make deductive assertions to an indicator of theories that make deductive or probabilistic assertions. Flew introduces the falsifiability challenge because, although theistic statements prima facie make assertions, many theists use common words in an uncommon way, thereby weakening the theistic statements. Flew's challenge is accordingly a question about the personal beliefs of his fellow disputants, and the goal is to elucidate what assertions the theistic statements make according to them, and accordingly what the words 'create' and 'love' mean according to them when it comes to God. It is an interesting psychological point that it may be easier for humans to identify a theory's assertions when they are asked to think about what the theory denies rather then what it asserts. But as far as a statement is independent of the vagaries of the human psyche, such a reformulation is not necessary. If the question is whether a statement makes assertions, it is enough to find a criterion that determines just that.

That a statement makes an assertion only if it makes an observational assertion is a very controversial assumption, but as Nielsen's consideration shows, some restriction to the assertions is necessary to arrive at a non-trivial challenge. Since according to some, theism is not meant to make observational assertions (cf. Diamond 1975), theism may not have to meet any challenge that demands them. ID, on the other hand, is meant to be an empirical theory, and thus has to make observational assertions.

The criterion for deductive observational assertions is simple (Lutz 2010, \$9.2):

Definition 1. A theory $H$ makes deductive observational assertions if and only if there are honest auxiliary assumptions $A$ and an observation statement $O$ such that

$$
H \wedge A \vDash O \text { and } A \not \models O \text {. }
$$


The definition differs from one given by Sober $(2008,151)$ only in its restriction to honest auxiliary assumptions. A set $A$ of auxiliary assumptions is honest if and only if every $S \in A$ is a justified statement, and $A$ also contains every statement on which the justification of $S$ depends. The use of auxiliary assumptions is necessary for the criterion's application to scientific theories, as Sober $(2007,5)$ argues, because scientific theories, "on their own, do not make testable predictions". ${ }^{3}$ But the auxiliary assumptions must not be arbitrary, for otherwise any theory $H$ would assert any statement $S$ with the help of auxiliary assumption $H \rightarrow S$. The auxiliary assumptions must be in some sense "suitable" (Sober 2008, 144). Sober's restrictions on the auxiliary assumptions allow a trivialization proof of his definition, but the trivialization can be avoided by demanding that the auxiliary assumptions be honest (Lutz 2010, \$9.2).

A criterion for probabilistic assertions (Lutz 2011b, $\$ 5.2$ ) is given by

Definition 2. A theory $H$ makes probabilistic observational assertions if and only if there are honest auxiliary assumptions $A$ and an observation statement $O$ such that

$$
\operatorname{Pr}(O \mid H \wedge A) \neq \operatorname{Pr}(O \mid A) .
$$

$\operatorname{Pr}(O \mid H \wedge A)$ is called the likelihood of $H$ (for $O$ ). Note that definition 2 contains definition 1 as a special case: Whenever $B \vDash C$ if and only if $\operatorname{Pr}(C \mid B)=1$ for all $B$ and $C$, inequality (2) is true if and only if $H \wedge A \vDash O$ and $A \not \models O .^{4}$

The inequality (2) is meant to have a truth value even if one or both of the occurring terms are undefined: If both terms are undefined, the inequality is false, if only one of them is undefined, the inequality is true (Lutz 2011b, \$5.2). Thus, given the auxiliary assumptions $A, H$ makes probabilistic observational assertions if and only if for some $O$, assuming $H$ influences the probability of $O$ or influences whether $O$ can be assigned a probability at all. In contradistinction, Sober $(2008,130)$ states that "a testable statement makes predictions, either by deductively entailing that an observation will occur or by conferring a probability on an observational outcome." While definition 1 captures the first disjunct of this claim, there is one kind of case in which definition 2 goes against the second disjunct: If assuming $H$ makes it impossible to assign a probability to an observation statement that otherwise would be assigned a probability by the auxiliary assumptions, definition 2 entails that $H$ makes assertions. And, pace Sober, it is very plausible that for a theory $H$ that makes no predictions, $\operatorname{Pr}(O \mid H \wedge A)$ is defined whenever $\operatorname{Pr}(O \mid A)$ is defined. For if $\operatorname{Pr}(O \mid A)$ is defined, then, given $A$, one must expect a specific regularity of occurrences of $O$. If $\operatorname{Pr}(O \mid H \wedge A)$ is then undefined, one must expect a breakdown of this regularity, and this expectation is plausibly an assertion. An example would be the assertion that under specific circumstances, some law fails that was assumed to hold universally. It certainly is pragmatically relevant when some observation statement can, contrary to the auxiliary assumptions, not be assigned a probability. ${ }^{5}$

With definitions 1 and 2, the falsifiability challenge can be modified to allow for probabilistic theories without giving up on its basic idea: The challenge for ID is to

\footnotetext{
${ }^{3}$ Since Sober does not use 'prediction' only to refer to claims about the future, I take it to be synonymous with 'assertion'.

${ }^{4}$ This argument can be made more precise and much longer (cf. Lutz 2011b, claim 8).

${ }^{5}$ In decision theory, this is acknowledged by the distinction between decisions under risk and decisions under ignorance.
} 
make observational assertions (simpliciter), that is, either deductive or probabilistic ones. This challenge is justified because it is plausible that every empirical theory must make some observational assertions. Laudan $(1983,37)$ claims to have given a counterexample of a respectable scientific theory that makes no observational assertions, but the relevant passage of the article he refers to (Laudan 1982, 17) contains only the point that theories often rely on auxiliary assumptions for their observational assertions. Since definitions 1 and 2 allow for auxiliary assumptions, this is not an objection to the modified falsifiability challenge.

Ayer $(1936,97)$ argues that "the purpose" of an empirical theory is "to enable us to anticipate the course of our sensations", so that making observational assertions is a necessary and sufficient condition for empirical theories. Popper sometimes even seems to take deductive observational assertions to be a necessary and sufficient condition (cf. Hansson 2008, $\$ 4.2)$. As Laudan $(1982,18)$ and Rothbart $(1982,95)$ note, this is clearly too strong. 'Anne got her head stuck in a drainpipe' makes an observational assertion, but is no scientific theory in that the statement does not, for example, offer the systematic economy or heuristic fertility that Hempel (1965) expects from a theory. This feature of scientific theories also poses a problem for the translatability challenge.

\section{The translatability challenge}

Tooley $(1975,489 f)$ provides both the name 'translatability challenge' and its most explicit formulation (the formulation by Ayer (1936, 114-120), although the basis of Tooley's, is comparatively freewheeling). Assuming that only the set of analytic statements is suitable as the set $A$ of auxiliary assumptions, the argument is roughly as follows: Only a statement that makes deductive observational assertions according to definition 1 has cognitive content, and two statements that in conjunction with $A$ entail the same observation statements have the same cognitive content. It follows that if a theological statement $T$ has cognitive content, the set $\Omega$ of observation statements entailed by $T \wedge A$ has some, namely the same cognitive content as $T$. Since the term 'God' in any theological statement $T$ is intended to refer to something that is not observational, 'God' does not occur in $\Omega$. Therefore a theological statement $T$ either has no cognitive content at all, or the specific theological content of $T$, the reference to God, is not cognitive.

There are a number of problems with this argument, for example the apparent irrelevance of $T$ having any cognitive content, and the problem of explicating the notion of 'reference'. I want to restrict my discussion to the simple point that it is doubtful that two theories that entail the same observation statements have the same cognitive content. For one, the theories may assign different probabilities to some observation statement without entailing it. ${ }^{6}$ Furthermore, it is doubtful that $T \wedge A$ can be replaced by $\Omega$ without cognitive loss. Hempel $(1965,222)$, for example, argues that non-observational statements are necessary for "inductive explanatory and predictive use and $[\ldots]$ systematic economy and heuristic fertility". Niiniluoto

\footnotetext{
${ }^{6}$ As with the falsifiability challenge, Tooley $(1975,505 f)$ uses an intentionally underspecified notion of testability to account for probabilistic inferences. And as in the case of the falsifiability challenge, this confuses the indicator of a property (being tested by the same observation statements) with the property itself (making the same observational assertions).
} 
(1972) and Tuomela (1973) give overviews of the discussion about the need for nonobservational statements, essentially supporting Hempel's conclusion. Thus there is no reason why theism would have to meet the translatability challenge, because it is implausible that a theory can be replaced without significant loss by the observation statements it entails.

On the other hand, a theory may be replaced by another theory without losing the theoretical virtues that can come with the use of non-observational statements. Such a replacement clearly comes with a clear cognitive loss, however, if the replaced theory is not disconfirmed and makes all the observational assertions of its replacement, while the replacing theory does not make all the observational assertions of the replaced theory. For then, the replacement would result in the systematization of fewer observations. Rothbart $(1982,99)$ suggests that a necessary condition for being scientific is to be worthy of experimental testing, which entails that a "hypothesis must encapsulate its rival's successes". Taking the successes to be the observational assertions of a theory that has not been disconfirmed, this is the modified translatability challenge.

Many contemporary theistic theories do not construe God's existence as competing with any empirical theories (cf. Mackie 1982, $\$ 8$; Sobel 2004, $\$$ VII), so that they are not intended as replacements. ID, on the other hand, is meant to replace ET, and since ET is not disconfirmed, this is not possible if ET makes all the observational assertions of ID, while ID fails to make all the observational assertions of ET.

This modification of the translatability challenge does not rely on any controversial assumptions about the cognitive content of theories, but does need a concept of 'making all the observational assertions of'. Deductively, this can be expressed by

Definition 3. Theory $H_{1}$ makes all the deductive observational assertions of $\mathrm{H}_{2}$ if and only if for all honest auxiliary assumptions $A$ and all observation statements $O$,

$$
H_{2} \wedge A \vDash O \text { only if } H_{1} \wedge A \vDash O \text {. }
$$

Condition (3) can be reformulated as ' $H_{2} \wedge A \vDash O$ only if either $A \vDash O$ or $H_{1} \wedge$ $A \vDash O$ ', which has the propositional structure ' $B \rightarrow(C \vee D)$ ', or, equivalently, ' $(B \rightarrow C) \vee(B \leftrightarrow D)$ '. Since furthermore $A \vDash O$ only if $H_{2} \wedge A \vDash O$ (i. e., ' $C \rightarrow B$ '), condition (3) amounts to

Either $H_{2} \wedge A \vDash O$ if and only if $A \vDash O$, or $H_{2} \wedge A \vDash O$ if and only if $H_{1} \wedge A \vDash O$.

This suggests

Definition 4. Theory $H_{1}$ makes all the probabilistic observational assertions of $H_{2}$ if and only if for all honest auxiliary assumptions $A$ and all observation statements $O$,

$$
\operatorname{Pr}\left(O \mid H_{2} \wedge A\right)=\operatorname{Pr}(O \mid A) \text { or } \operatorname{Pr}\left(O \mid H_{2} \wedge A\right)=\operatorname{Pr}\left(O \mid H_{1} \wedge A\right) .
$$

As can be seen from formula (4), definition 4 and definition 3 coincide whenever $B \vDash C$ if and only if $\operatorname{Pr}(C \mid B)=1$. With these definitions, $H_{1}$ can be said to make all the observational assertions (simpliciter) of $\mathrm{H}_{2}$ if and only if it makes all the deductive and probabilistic observational assertions of $\mathrm{H}_{2}$. It is easily shown that if a theory $H$ makes no observational assertions, all other theories, and specifically tautologies, 
make all the observational assertions of $H .^{7}$ Since further $H$ does not make all observational assertions of a theory $H^{\prime}$ that makes any, $H$ fails the translatability challenge against any such $H^{\prime}$ that is not disconfirmed.

The modified translatability challenge is harder to meet than the modified falsifiability challenge, and differs fundamentally in its structure. ID is not evaluated on its own, but rather compared to ET, the theory it is meant to replace. To meet the translatability challenge against ET, ID must account for all those observations that ET can already account for.

\section{The testability challenge}

Like Tooley, Sober (1990; 1999; 2007; 2008, \$2) modifies the falsifiability challenge by generalizing falsifiability to testability. The main difference to Tooley's approach is that Sober gives a definition of 'testing'. The testing of deductive theories is simple (Sober 2008, 52, n. 29):

If a true observation sentence entails $H[\ldots]$ you can conclude without further ado that $H$ is true; this is just modus ponens. And if $H$ entails $O$ and $O$ turns out to be false, you can conclude that $H$ is false [..]; this is just modus tollens.

Taking into account the need for auxiliary assumptions, this leads to

Definition 5. Theory $H$ is deductively testable if and only if there are honest auxiliary assumptions $A$ and an observation statement $O$ such that

$$
O \wedge A \vDash \neg H \text { or } O \wedge A \vDash H \text {. }
$$

Note that Sober here already changes the challenge, since definition 5 is not equivalent to definition 1: According to Sober, a theory is deductively testable if and only if it is falsifiable or verifiable.

The testing of theories through their probabilistic assertions is not a straightforward generalization of the testing of theories through their deductive assertions, Sober $(2008, \$ 1.2)$ contents, since a theory is not always improbable if it assigns a low probability to a true observation statement. Rather, an observation statement can only test one theory relative to another, namely if it is assigned different probabilities by the two theories (Sober 2008, $\mathbb{\$} 1.3$ ). Considering the need for auxiliary assumptions, Sober $(2008,152)$ suggests the following definition:

Hypothesis $H_{1}$ can now be tested against hypothesis $H_{2}$ if and only if there exist true auxiliary assumptions $A$ and an observation statement $O$ such that (i) $\operatorname{Pr}\left(O \mid H_{1} \wedge A\right) \neq \operatorname{Pr}\left(O \mid H_{2} \wedge A\right)$, (ii) we now are justified in believing $A$, and (iii) the justification we now have for believing $A$ does not depend on believing that $H_{1}$ is true or that $H_{2}$ is true and also does not depend on believing that $O$ is true (or that it is false).

\footnotetext{
${ }^{7}$ It is also easily shown that two theories are empirically equivalent (Lutz 2011b, def. 11) if and only if each theory makes all the observational assertions of the other.
} 
As in Sober's criterion for theories that make deductive observational assertions, the restrictions on the auxiliary assumptions are too weak, allowing a trivialization proof. The trivialization proof can again be blocked by demanding that the auxiliary assumptions be honest (Lutz 2011a, \$4.3). Furthermore, Sober has an idiosyncratic interpretation of the inequality (i) (Lutz 2011a, \$3.1), which leads to

Definition 6 (Contrastive testability). Theory $H_{1}$ can be tested against theory $H_{2}$ if and only if there are honest auxiliary assumptions $A$ and an observation statement $O$ such that $\operatorname{Pr}\left(O \mid H_{1} \wedge A\right)$ and $\operatorname{Pr}\left(O \mid H_{2} \wedge A\right)$ are defined and

$$
\operatorname{Pr}\left(O \mid H_{1} \wedge A\right) \neq \operatorname{Pr}\left(O \mid H_{2} \wedge A\right) .
$$

A theory is then testable (simpliciter) if and only if it is deductively or contrastively testable, and the challenge is to show that ID is deductively testable or contrastively testable against ET.

What role contrastive testability could play in a criticism of ID is somewhat unclear, because the definition is symmetric: The definiens is invariant up to logical equivalence under exchange of $H_{1}$ and $H_{2}$. If ID turned out not to be testable against ET, this would therefore be prima facie bad for ID if and only if it would be bad for ET. ID may have further properties that distinguish it negatively from ET, but the question would then still be what role contrastive testability can play. Of course, that question only has to be answered if ID is in fact not contrastively testable against ET.

\section{The testability of ID}

To show that ID cannot meet the testability challenge, Sober $(2007,3)$ considers a minimal version of ID:

The single thesis of what I will call mini-ID is that the complex adaptations that organisms display (e. g., the vertebrate eye) were crafted by an intelligent designer.

Sober's general strategy is to argue that ID cannot be tested against ET because ID "does not predict much of anything" (Sober 2008, \$2.15). I will consider this argument in $\$ 5.2$. Surprisingly, Sober first argues that ID is deductively testable.

\subsection{The deductive testability of ID}

Although the designer in ID is not specified and thus has wholly mysterious intentions (Sober 1999, 65; Sober 2007, 6; Sober 2008, 128, n. 14), Hartwig and Meyer (1993, $160)$ argue that ID is still falsifiable. For "the concept of intelligent design predicts that complex information, such as that encoded in a functioning genome, never arises from purely chemical or physical antecedents". Against this, Sober $(2007,6,5 ; 2008$, 130) first points out that probabilistic theories are not falsifiable and falsifiability therefore cannot be a good criterion of testability. Second, implicitly assuming that a not purely chemical or physical antecedent is always an intelligent designer, he argues that the argument is invalid because the statement 'Somewhere on the causal chain leading up to "complex information" there is an intelligent designer at work' is not falsifiable. This is because 'somewhere' may refer to a point outside of space and time, 
and because the intelligent designer may be unobservable, so that ID does not make an observational assertion (Sober 2007, 6f).

The second point is well-taken, and echos Nielsen's remark that every theory is falsifiable if it only has to be incompatible with some statement. A falsifiable theory must be incompatible with an observation statement, and that something "causes" or (metaphorically) "arises from" something else is not observational. Even if it usually was, the antecedents that cause complex information, or from which complex information arises, can be unobservable; they could be God or some other unobservable designer, for example. And the statement that some two-place relation holds is not generally observational if one of the relation's arguments is not observable. This is especially clear for an otherwise observational relation like 'is standing next to'. However, the point is also a red herring because Sober (2008, 148; cf. 2008, 130; 2007, 4) himself considers it "perfectly clear" that ID deductively entails the observation statement 'Organisms display complex adaptations' ('ADAP' in the following), so that ID is falsifiable. Hartwig and Meyer have only chosen the wrong statement to argue their case.

This is important because Sober's first point, his dismissal of falsifiability, is mistaken according to his own claims: While falsifiability is not a necessary condition for deductive testability, it is, according to definition 5, a sufficient condition. This is not an uncharitable interpretation of Sober's position, for Sober $(1999,72, \mathrm{n} .14)$ states:

The thesis that testing is contrastive requires that prediction be probabilistic; otherwise, hypotheses could be falsified without any contrastive alternative having to play a role. If $H \& A$ deductively entails $O$, and $A$ is known to be true, then, if we observe not- $O$, we can conclude that $H$ is false.

And in a passage already referred to, Sober (2008, 52, n. 29) states:

There are two exceptions to the thesis that testing is always contrastive. If a true observation statement entails $H$, there is no need to consider alternatives to $H$; you can conclude without further ado that $H$ is true; this is just modus ponens. And if $H$ entails $O$ and $O$ turns out to be false, you can conclude that $H$ is false, again without needing to contemplate alternatives; this is just modus tollens.

Since ID entails ADAP, it is falsifiable and thus deductively testable. Therefore, it is also testable simpliciter.

\subsection{The contrastive testability of ID}

Sober $(2008, \$ 2.12)$ argues that the "problem with the hypothesis of intelligent design" is that "it doesn't predict much of anything" (Sober 2008, \$2.15). (The qualifier 'much of' is necessary because according to Sober, ID does assert that organisms display complex adaptations.) And since for "ID to be testable, it must make predictions" (Sober 2007, 7), "the design hypothesis is untestable" (Sober 2008, 148; cf. Sober 1999, 66f). This conclusion is false because ID is falsifiable, so that Sober's claim at least has to be weakened to the claim that ID is not contrastively testable against ET. I will now argue that ID is contrastively testable against ET as well. 
First, note that Sober's argument that ID cannot be tested against ET relies on a hidden premise: Sober claims that ID does not make observational assertions besides ADAP, so that $\operatorname{Pr}(O \mid \operatorname{ID} \wedge A)=\operatorname{Pr}(O \mid A)$ whenever both probabilities are defined and $O \neq$ ADAP. Furthermore, if ID cannot be tested against ET, $\operatorname{Pr}(O \mid$ ET $\wedge A)=$ $\operatorname{Pr}(O \mid \operatorname{ID} \wedge A)$ whenever both probabilities are defined. Therefore, Sober's argument is valid only if for any $O \neq \mathrm{ADAP}$ such that $\operatorname{Pr}(O \mid$ ET $\wedge A) \neq \operatorname{Pr}(O \mid A)$ and both probabilities are defined, $\operatorname{Pr}(O \mid \mathrm{ID} \wedge A)$ is undefined. But ET makes predictions, and, more specifically, there are many $O^{\prime} \neq$ ADAP such that $\operatorname{Pr}\left(O^{\prime} \mid\right.$ ET $\left.\wedge A\right) \neq \operatorname{Pr}\left(O^{\prime} \mid A\right)$ and both probabilities are defined. Then, since $\operatorname{Pr}(O \mid A)$ is defined, by definition 2 $\operatorname{Pr}\left(O^{\prime} \mid \operatorname{ID} \wedge A\right)$ is defined as well. Thus the hidden premise is false. Furthermore, since $\operatorname{Pr}\left(O^{\prime} \mid\right.$ ET $\left.\wedge A\right) \neq \operatorname{Pr}\left(O^{\prime} \mid A\right)=\operatorname{Pr}\left(O^{\prime} \mid \operatorname{ID} \wedge A\right)$, where all probabilities are defined, ID can be tested against ET.

This inference relies on the possibly contentious interpretation of inequality (2) according to which, if ID makes no probabilistic assertions, $\operatorname{Pr}(O \mid \operatorname{ID} \wedge A)$ is always defined when $\operatorname{Pr}(O \mid A)$ is defined. I will now argue that even under a weaker assumption, ID can be tested against ET. That $\operatorname{Pr}(O \mid \mathrm{ID} \wedge A)$ is at least sometimes defined when $\operatorname{Pr}(O \mid A)$ is defined follows from an example that Sober $(1999, \mathrm{n} .24)$ attributes, in a different context, to Greg Mougin:

Let $H_{1}=$ God created the eye, $E=$ Jones is pregnant, $A=$ Jones is sexually active, and $H_{2}=$ Jones used birth control. It is possible to test $H_{1}$ against $H_{2}$; given independently attested background assumptions $A, E$ favors $H_{1}$ over $H_{2}$. The reason is that $\operatorname{Pr}(E \mid A)=\operatorname{Pr}\left(E \mid A \& H_{1}\right)>$ $\operatorname{Pr}\left(E \mid A \& H_{2}\right)$.

Since for Sober $(1999,62,65), H_{1}$ here stands for ID, $\operatorname{Pr}(O \mid \operatorname{ID} \wedge A)$ is defined for some observation $O \neq \mathrm{ADAP}$, for example that Jones is pregnant. It is thus not clear why, for all $O \neq$ ADAP with $\operatorname{Pr}(O \mid$ ET $\wedge A) \neq \operatorname{Pr}(O \mid A)$, ID should render the probability for $O$ undefined.

It is thus very plausible that at least for one $O^{*} \neq \mathrm{ADAP}$ for which $\operatorname{Pr}\left(O^{*} \mid \mathrm{ET} \wedge A\right) \neq$ $\operatorname{Pr}\left(O^{*} \mid A\right)$ and both probabilities are defined, $\operatorname{Pr}\left(O^{*} \mid \operatorname{ID} \wedge A\right)$ is defined as well. This very weak assumption is also independently plausible: Take the statement that the eye of some organism has a specific feature. Based on our background knowledge about the ratio of the occurrence of this feature in other organisms' eyes, we might be able to assign a specific probability $p$ to the occurrence of this feature, and based on our background knowledge and ET, we might be able to assign a different probability. But based on our background knowledge, we can also infer that if that eye was created by a designer, this designer had the intention and ability to create an eye with that feature with probability $p$. In conjunction with ID, the probability for the occurrence of the feature then does not become undefined, but rather keeps the value $p$.

Under this very weak assumption, ID can be tested against ET. For then $\operatorname{Pr}\left(O^{*} \mid\right.$ ET $\left.\wedge A\right) \neq \operatorname{Pr}\left(O^{*} \mid A\right)=\operatorname{Pr}\left(O^{*} \mid \operatorname{ID} \wedge A\right)$, where all probabilities are defined, so that ID can be tested against ET.

\section{The three challenges to intelligent design}

I have not argued that Sober is correct in his claim that ID makes no observational assertion besides ADAP, but have only analyzed the implications of this claim. Under 
this assumption, ID meets the modified falsifiability challenge. However, ID fails the modified translatability challenge when compared to any other theory that entails ADAP and makes any other observational assertion. One such theory is, according to Sober, ET. That according to Sober ID makes an observational assertion is thus not a decisive argument in favor of ID. It just means that ID meets one of two challenges.

Sober suggests contrastive testability as an improvement over falsifiability, and argues that ID is not contrastively testable because it does not assert "much of anything". This all sounds as if he claimed that ID fails to meet a modification of the falsifiability challenge. However, when it comes to the observational assertion that organisms display complex adaptations, Sober argues that ET makes the same assertion, and thus ID is still not contrastively testable. This sounds as if Sober claimed that ET fails to meet the modified translatability challenge. A theory that fails to meet the modified falsifiability challenge indeed fails the modified translatability challenge against almost any other theory, but a theory that makes almost no observational assertions still makes some.

Sober presumably treats the three challenges as one because he assumes that a theory that does not make observational assertions renders all likelihoods undefined. It is surprising that Sober never argues for this claim. Since it is false, it is unsurprising that it leads to inconsistencies. Given such confusions, and since it is not clear how a symmetric relation between theories can provide a reason to prefer one theory over another, it seems irrelevant whether ID meets the testability challenge against ET.

Whether ID meets the modified falsifiability or translatability challenge depends on whether Sober is right in arguing that ID makes only one observational assertion, and right in his exposition of ID. Sober claims that 'The complex adaptations were crafted by an intelligent designer' and 'The complex adaptations are the result of natural selection' both entail ADAP. But, it seems, so does 'The complex adaptations are the result of circles being round', which gives Sober's inference the air of hocuspocus. Thus, while the status of the three criteria is reasonably clear, the status of ID still requires investigation.

\section{References}

Ayer, A. J. (1936). Language, Truth and Logic. Victor Gollanz, London, $1^{\text {st }}$ edition. References are to the second edition (Ayer 1946).

Ayer, A. J. (1946). Language, Truth and Logic. Victor Gollanz, London, $2^{\text {nd }}$ edition.

Diamond, M. L. (1975). Verificationism: Difficulties and proposals. In Diamond and Litzenburg (1975), pages 435-445.

Diamond, M. L. and Litzenburg, T. V., editors (1975). The Logic of God: Theology and Verification. Bobbs-Merill, Indianapolis, IN.

Flew, A. (1950). Theology and falsification. University, 1:1-8. References are to the reprint (Diamond and Litzenburg 1975, 257-259).

Flew, A. (1975). "Theology and falsification" in retrospect. In Diamond and Litzenburg (1975), pages 269-283. 
Hansson, S. O. (2008). Science and pseudo-science. In Zalta, E. N., editor, The Stanford Encyclopedia of Philosophy. The Metaphysics Research Lab, Center for the Study of Language and Information, Stanford University, Stanford, fall 2008 edition.

Hartwig, M. D. and Meyer, S. C. (1993). A note to teachers. In Of Pandas and People: The Central Question of Biological Origins, pages 153-164. Foundation for Thought and Ethics, Richardson, TX, $2^{\text {nd }}$ edition.

Hempel, C. G. (1965). The theoretician's dilemma. In Aspects of Scientific Explanation and Other Essays in the Philosophy of Science, pages 173-226. The Free Press, New York.

Laudan, L. (1982). Commentary: Science at the bar-causes for concern. Science, Technology, E Human Values, 7(41):16-19.

Laudan, L. (1983). More on creationism. Science, Technology, E Human Values, 8(1):36-38.

Lutz, S. (2010). Criteria of empirical significance: A success story. Preprint: http: //philsci-archive.pitt.edu/8423/. Forthcoming.

Lutz, S. (2011a). On a contrastive criterion of testability I: Defining contrastive testability. Preprint: http://philsci-archive.pitt.edu/8574/. Forthcoming.

Lutz, S. (2011b). On a contrastive criterion of testability II: The material inadequacy of contrastive testability. Preprint: http://philsci-archive.pitt.edu/8575/. Forthcoming.

Mackie, J. L. (1982). The Miracle of Theism. Arguments for and against the Existence of God. Clarendon Press, Oxford.

Nielsen, K. (1966). On fixing the reference range of 'God'. Religious Studies, 2(1):1336.

Niiniluoto, I. (1972). Inductive systematization: Definition and a critical survey. Synthese, 25(1-2).

Paley, W. (1802). Natural Theology: Or, Evidences of the Existence and Attributes of the Deity. Collected From the Appearances of Nature. R. Foulder, London. References are to the reprint (Paley 1830).

Paley, W. (1830). Natural Theology: Or, Evidences of the Existence and Attributes of the Deity. Collected From the Appearances of Nature. Hilliard and Brown, Cambridge.

Rothbart, D. (1982). Demarcating genuine science from pseudoscience. In Grim, P., editor, Philosophy of Science and the Occult, SUNY Series in Philosophy. State University of New York Press, Albany.

Sobel, J. H. (2004). Logic and Theism. Arguments For and Against Beliefs in God. Cambridge University Press, Cambridge. 
Sober, E. (1990). Contrastive empiricism. In Savage, C. W., editor, Scientific Theories, volume 14 of Minnesota Studies in the Philosophy of Science, pages 392-410. University of Minnesota Press, Minneapolis, MN.

Sober, E. (1999). Testability. Proceedings and Addresses of the American Philosophical Association, 73(2):47-76.

Sober, E. (2007). What is wrong with intelligent design? The Quarterly Review of Biology, 82(1):3-8.

Sober, E. (2008). Evidence and Evolution: The Logic Behind the Science. Cambridge University Press, Cambridge.

Swinburne, R. (2004). The Existence of God. Clarendon Press, Oxford, $2^{\text {nd }}$ edition.

Tooley, M. (1975). Theological statements and the question of an empiricist criterion of cognitive significance. In Diamond and Litzenburg (1975), pages 481-524.

Tuomela, R. (1973). Theoretical Concepts. Number 10 in Library of Exact Philosophy. Springer, Wien. 